\title{
Advanced secondary abdominal pregnancy: A complication of induced abortion
}

\author{
Fateme Lalooha, Khadijeh Elmizadeh, Fateme Salehi
}

\begin{abstract}
Introduction: Secondary abdominal pregnancies usually develop as a result of a tubal abortion, tubal rupture or uterine rupture with intraabdominal implantation. Case Report: We report a 40-year-old patient gravida IV, para III suffering from abdominal pain. She was in the 29th week of gestation according to a 14thweek normal sonogram. She had unsuccessfully attempted to induce abortion with the assistance of a home midwife in the 14th week. This caused severe abdominal pain, but she chose not to be hospitalized because of fear of prosecution. Ultrasound demonstrated that the fetus was out of the uterus and revealed a heterogeneous mass in the left lower quadrant. Laparotomy revealed an area of thin fibrotic scar behind the uterus with normal tubes and ovaries. The fetus in the amniotic sac was surrounded by intestinal loops. The placenta, implanted behind the left broad ligament at the edge of the scar area was removed with a brisk bleeding, which was controlled. No postoperative complications were observed. Conclusion: Presence of little free fluid and a uterine scar with normal fallopian tubes against a history of an unsuccessful induced abortion and severe illness are highly suggestive of a uterine rupture resulting in secondary implantation on
\end{abstract}

Fateme Lalooha1, Khadijeh Elmizadeh ${ }^{1}$, Fateme Salehi ${ }^{2}$ Affiliations: ${ }^{1}$ Obstetrician and Gynecologist, Department of Obstetrics \& Gynecology, Qazvin University of Medical Science, Qazvin, Iran; ${ }^{2}$ Resident of Obstetrics and Gynecology, Department of Obstetrics \& Gynecology, Qazvin University of Medical Science, Qazvin, Iran.

Corresponding Author: Khadijeh Elmizadeh, Qazvin, Iran 3415646139; Ph: 00989121813620; Fax: 00982812236378;

Email ID: parisa_elmizadeh@yahoo.com

Received: 04 September 2012

Accepted: 23 February 2013

Published: 01 September 2013 the broad ligament. This is probably the first case that shows an abdominal pregnancy can result from unsuccessful induced abortion. In the countries where abortion is not permitted, patients with low socioeconomic status are prone to the dangerous consequences of illegal attempts to induce abortion. Close observation and special follow-up care are necessary in such cases.

Keywords: Induced Abortion, Pregnancy, Secondary Abdominal Pregnancy

$$
* * * * * * * * *
$$

Lalooha F, Elmizadeh K, Salehi F. Advanced secondary abdominal pregnancy: A complication of induced abortion. International Journal of Case Reports and Images 2013;4(9):494-497.

$$
* * * * * * * * *
$$

doi:10.5348/ijcri-2013-09-364-CR-8

\section{INTRODUCTION}

Abdominal pregnancy is a rare event and is classified as either primary or secondary, with the latter being more common. Secondary abdominal pregnancies usually develop as a result of a tubal abortion, tubal rupture, or uterine rupture with intra-abdominal implantation [1].

\section{CASE REPORT}

A 40-year-old gravida IV, para III woman was referred to us in the 29th week of gestation with a complaint of a two-week-long abdominal pain and weakness. The accompanying symptoms were vertigo and loss of appetite. There were signs of anemia. Her vital signs were stable. She had lost $3 \mathrm{~kg}$ in two weeks. Pregnancy had been confirmed three months earlier through serum 
hCG measurement followed by ultrasound examination showing a normal 14-week pregnancy with antero fundal placenta. She reported no remarkable medical and surgical history and claimed that her early pregnancy was uneventful.

Physical examination upon admission revealed an abdominal mass rising from the pelvis without tenderness, rebound, and guarding. Laboratory results showed hemoglobin level $7.7 \mathrm{~g} / \mathrm{dL}$ and a hematocrit value 25.7. Other examinations, including $\mathrm{BUN}, \mathrm{Cr}$, serum electrolytes, coagulation, and liver function tests, showed were normal. Gestational age was 29 weeks +4 days according to 14 weeks sonogram. However, ultrasonographic scan confirmed a breech-lying 26 weeks +2 days normal fetus out of the uterus with oligohydramnios and a vague heterogeneous $157 \times 79 \mathrm{~mm}$ mass in the left lower quadrant with some free fluid in the abdominal cavity. The uterus was empty and normally placed. The MRI scan confirmed the diagnosis (Figure 1).

After the diagnosis was explained to the patient and her family, they revealed a history of an unsuccessful attempt to induce abortion with the help of a home midwife. This attempt caused severe illness, paleness and crampy abdominal pain, which wore off after two weeks. Despite severe illness, she had not visited any physician because of fear of prosecution.

The patient was given four units of packed cell which increased her hemoglobin to $11.7 \mathrm{~g} / \mathrm{dL}$. She underwent bowel preparation prior to the surgery. Accessibility of sufficient blood products was confirmed. A team consisting of two obstetricians/gynecologists, a general surgeon, a vascular surgeon and an anesthesiologist prepared for laparotomy under general anesthesia. The parietal peritoneum was opened through a midline

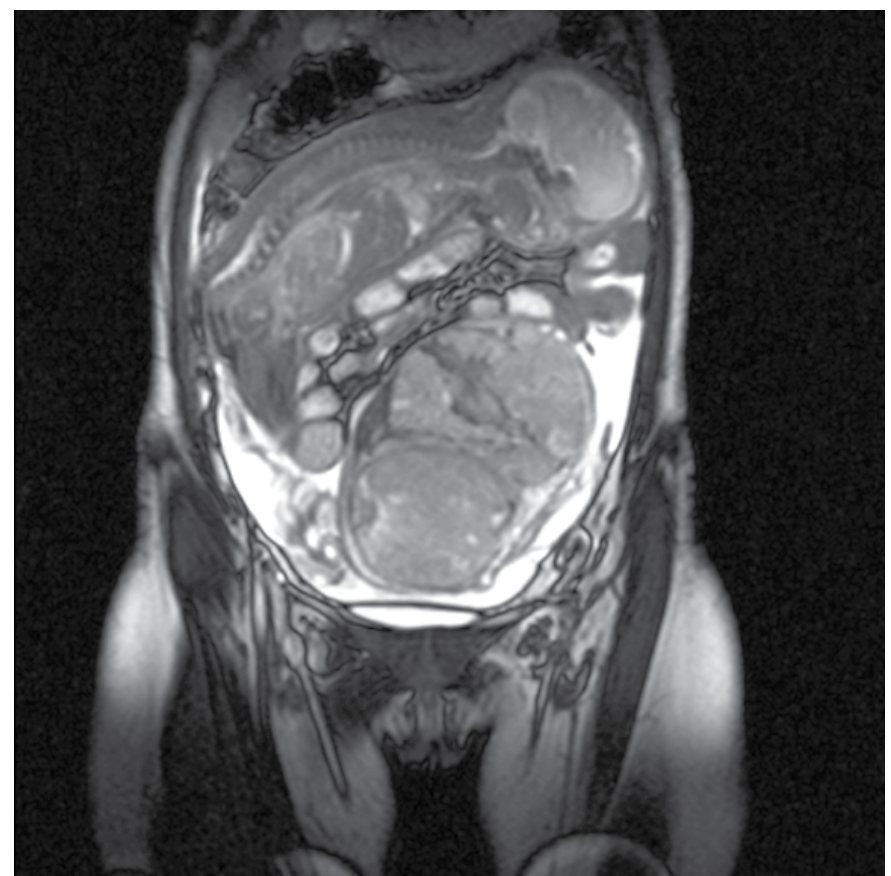

Figure 1: Magnetic resonance imaging showing uterus, fetus, and placenta. incision. The amniotic sac was surrounded by intestinal loops. The baby was delivered. She had no dysmorphic features and weighed $800 \mathrm{~g}$ with an Apgar score of 4-O at 1-20 minutes. The amniotic membranes were loosely clinging to the loops of jejunum and ileum and were easily dissected away. The uterus, fallopian tubes, and ovaries were normal. The placenta was implanted behind the uterus, on the left broad ligament and the culde-sac. There was a thin fibrotic scar measuring $3 \times 2 \mathrm{~cm}$ behind the uterus near the edge of the placenta and was only covered by uterine serosa. Attempting to remove the placenta resulted in a brisk bleeding, but it was controlled by packing and suturing. The fallopian tubes were ligated, and the uterus was repaired. The patient was transfused with two units of packed cell during operation. No complications were observed after the surgery. The patient was discharged after six days in good health.

\section{DISCUSSION}

This case revealed the presence of little free fluid and a uterine scar with normal fallopian tubes. These findings when considered against backdrop of an unsuccessful induced abortion and severe illness are highly suggestive of a uterine rupture resulting in secondary implantation on the broad ligament.

In a review of available literature, we found only a few cases of secondary abdominal pregnancy resulting from uterine rupture. Teng et al. (2007) reported a secondary abdominal pregnancy following rupture of a uterine scar after two cesarean sections [2]. They claimed that their report was the first case of an early scar rupture resulting in a viable intra-abdominal pregnancy. Amritha et al. reported a viable secondary abdominal pregnancy following rupture of a rudimentary horn [3]. We can assume that our case is the first report of secondary abdominal pregnancy as a complication of an induced abortion.

Abdominal pregnancy causes relatively few symptoms, none of which are individually diagnostic [4]. Once she recovered from the acute symptoms of uterine damage, our patient was relatively asymptomatic in the rest of her pregnancy. Thus, a high index of suspicion is warranted for diagnosing abdominal pregnancy [3]. Ultrasonography is the main method for diagnosis $[5,6]$, but it fails to diagnose in half of the cases [4, 5]. The 29th-week sonogram of our patient revealed an abdominal pregnancy with the following findings: separation of the uterus from the fetus, extrauterine placenta, oligohydramnios, abnormal lie of the fetus, some free peritoneal fluid and poor visualization of the placenta.

An MRI scan confirmed all the sonographic findings. Thus, it can be a useful adjunct to the ultrasound and may help identifying the spread of placental implantation. It seems wise to administer both tests to obtain maximal information for surgical planning $[3,4]$. 
We chose to terminate the pregnancy upon diagnosis because a review of previous case reports showed that employing expectant management in the hope of fetal maturity involves a substantial risk of life-threatening hemorrhage $[4,5]$.

Fortunately, it was possible to separate the whole placenta intra-operatively. Placental management is a highly controversial issue. Retention of the placenta poses substantial risks. Most authors agree that the placenta should be removed provided its blood supply is identified and can be ligated without damaging other organs $[3,5]$.

Completely removal of the placenta made postoperative methotrexate administration unnecessary. Indeed, postoperative use of methotrexate is controversial in the case of the placenta left in place [4].

\section{CONCLUSION}

Abdominal pregnancy is a rare condition and diagnosis requires a high index of suspicion. In the countries where abortion is illegal, patients with a low socioeconomic status are prone to the dangerous consequences of secret attempts to induce abortion. So it is imperative that every primary health care provider closely observe the patients with unwanted pregnancy and provide special follow-up care. Prompt treatment upon discovery of the condition is essential. In managing abdominal pregnancy, maternal morbidity and mortality can be significantly reduced through preoperative diagnosis and evaluation, sufficient blood supply, bowel preparation, availability of a multidisciplinary surgical team and proper operative techniques.

\section{ACKNOWLEDGEMENTS}

Thanks Mr Mahmood Soleimani for his helpful commends in writing the case report.

$* * * * * * * * *$

\section{Author Contributions}

Fateme Lalooha - Substantial contributions to conception and design, Acquisition of data, Analysis and interpretation of data, Drafting the article, Revising it critically for important intellectual content, Final approval of the version to be published

Khadijeh Elmizadeh - Acquisition of data, Drafting the article, Revising it critically for important intellectual content, Final approval of the version to be published Fateme Salehi - Acquisition of data, Drafting the article, Revising it critically for important intellectual content, Final approval of the version to be published

\section{Guarantor}

The corresponding author is the guarantor of submission.

\section{Conflict of Interest}

Authors declare no conflict of interest.

\section{Copyright}

(C) Fatemeh Lalooha et al. 2013; This article is distributed under the terms of Creative Commons attribution 3.0 License which permits unrestricted use, distribution and reproduction in any means provided the original authors and original publisher are properly credited. (Please see www.ijcasereportsandimages.com/copyright-policy.php for more information.)

\section{REFERENCES}

1. Mahomed K. Abdominal Pain. In: James DK, Steer PJ, Weiner CP, Gonik B, Crowther CA\& Robson SC, editors. High Risk Pregnancy: Management Options. Philadelphia, Pennsylvania: Saunders 2010:1013-26.

2. Teng HC, Kumar G, Ramli NM. A viable secondary intra-abdominal pregnancy resulting from rupture of uterine scar: role of MRI. Br J Radiol 2007;80(955):e134-6.

3. Amritha B, Sumangali T, Priya B, Deepak S, Sharadha R. A rare case of term viable secondary abdominal pregnancy following rupture of a rudimentary horn: a case report. J Med Case Reports 2009;3:38-40.

4. Worley $\mathrm{KC}$, Hnat MD, Cunningham FG. Advanced extrauterine pregnancy: diagnostic and therapeutic challenges. Am J Obstet Gynecol 2008 Mar;198(3):297.e1-7.

5. Dahab AA, Aburass R, Shawkat W, Babgi R, Essa O, Mujallid RH. Full-term extrauterine abdominal pregnancy: a case report. J Med Case Reports 2011;5:531-4.

6. Yildizhan R, Kolusari A, Adali F, et al. Primary abdominal ectopic pregnancy: a case report. Cases J 2009;2:8485-9. 
Access PDF of article on other devices other devices

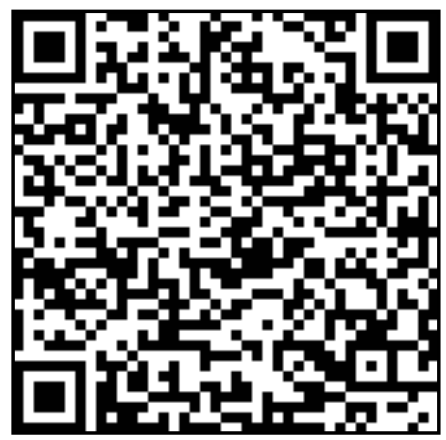

